\title{
Phaseless inverse scattering with background information
}

\section{R.G. Novikov ${ }^{1,2}$, V.N. Sivkin ${ }^{1,3}$}

${ }^{1}$ CMAP, CNRS, Ecole Polytechnique, Institut Polytechnique de Paris, 91128 Palaiseau, France

2 IEPT RAS, 117997 Moscow, Russia

${ }^{3}$ Lomonosov MSU, Moscow, 119991, Russia

Email: novikov@cmap.polytechnique.fr, sivkin96@yandex.ru,

Abstract. We consider phaseless inverse scattering for the multidimensional Schrödinger equation with unknown potential $v$ using the method of known background scatterers. In particular, in dimension $d \geq 2$, we show that $\left|f_{1}\right|^{2}$ at high energies uniquely determines $v$ via explicit formulas, where $f_{1}$ is the scattering amplitude for $v+w_{1}, w_{1}$ is an a priori known nonzero background scatterer, under the condition that supp $v$ and supp $w_{1}$ are sufficiently disjoint. If this condition is relaxed, then we give similar formulas for finding $v$ from $|f|^{2},\left|f_{1}\right|^{2}$, where $f$ is the scattering amplitude for $v$. In particular, we continue studies of [Novikov, J. Geom. Anal. 26(1), 346-359, 2016], [Leshem et al, Nature Communications 7(1), 1-6, 2016].

Keywords: Schrödinger equation, Helmholtz equation, phaseless inverse scattering, phase retrieval problem

AMS subject classification: 35J10, 35P25, 35R30, 81U40

\section{Introduction}

We consider the scattering problem for the stationary Schrödinger equation

$$
-\Delta \psi+v(x) \psi=E \psi, \quad x \in \mathbb{R}^{d}, d \geq 1, E>0,
$$

where $\Delta$ is the standard Laplacian in $x$,

$$
\begin{aligned}
& v \in L^{\infty}\left(\mathbb{R}^{d}\right), v \text { is complex-valued, supp } v \subset D, \\
& D \text { is an open bounded domain in } \mathbb{R}^{d} .
\end{aligned}
$$

The Schrödinger equation (1), under assumptions (2), describes a non-relativistic quantum mechanical particle at fixed energy $E$ interacting with a macroscopic object contained in $D$, where $v$ is the potential of this interaction (and we assume that $\frac{\hbar^{2}}{2 m}=1$ ).

Equation (1) at fixed $E$ can be also considered as the Helmholtz equation of electrodynamics or acoustics at fixed frequency $\omega$. In this context $v$ and $E$ are interpreted as follows:

$$
v(x)=\left(1-n^{2}(x)\right) E, \quad E=\left(\frac{\omega}{c_{0}}\right)^{2},
$$

where $n(x)$ is a scalar index of refraction, $n(x) \equiv 1$ on $\mathbb{R}^{d} \backslash D, c_{0}$ is a reference speed of wave propagation; and in the simplest case $n(x)=c_{0} / c(x)$, where $c(x)$ is a speed of wave propagation.

For equation (1) we consider the classical scattering solutions $\psi^{+}=\psi^{+}(x, k), x \in \mathbb{R}^{d}, k \in$ $\mathbb{R}^{d}, k^{2}=E$, specified by the following asymptotics as $|x| \rightarrow \infty$ :

$$
\psi^{+}(x, k)=e^{i k x}+\frac{e^{i|k||x|}}{|x|^{(d-1) / 2}} A\left(k,|k| \frac{x}{|x|}\right)+O\left(\frac{1}{|x|^{(d+1) / 2}}\right),
$$

for some a priori unknown $A$. The function $\psi^{+}=\psi^{+}(x, k)$ describes scattering of the incident plane waves described by $e^{i k x}$ on the scatterer described by $v$. The second term on the right-hand side of (4) describes the leading scattered spherical waves. The function $A$ arising with this term is the scattering amplitude for equation (1) for fixed $E$. This function is defined on

$$
\mathcal{M}_{E}=\left\{k, l \in \mathbb{R}^{d}: \quad k^{2}=l^{2}=E\right\}=S_{\sqrt{E}}^{d-1} \times S_{\sqrt{E}}^{d-1} .
$$


It is convenient to present $A$ as follows:

$$
\begin{gathered}
A(k, l)=c(d,|k|) f(k, l),(k, l) \in \mathcal{M}_{E}, \\
c(d,|k|)=-\pi i(-2 \pi i)^{(d-1) / 2}|k|^{(d-3) / 2}, \text { for } \sqrt{-2 \pi i}=\sqrt{2 \pi} e^{-i \pi / 4}
\end{gathered}
$$

see formulas (9), (13), (16). We also use the terminology "scattering amplitude" for $f$ arising in $(4),(6)$.

In order to study $\psi^{+}$and $f$ one can use the Lippmann-Schwinger integral equation (12) for $\psi^{+}$and the integral formula (13) for $f$; see Section 2 .

We recall that in quantum mechanics the complex (phased) values of the functions $\psi^{+}$and $f$ have no direct physical sense, whereas the phaseless values of $\left|\psi^{+}\right|^{2}$ and $|f|^{2}$ have probabilistic interpretation (the Born's principle) and can be obtained in experiments; see [6], [11]. On the other hand, in acoustics or electrodynamics the complex values of $\psi^{+}$and $f$ can be directly measured, at least, in principle. However, in many important cases of monochromatic electro-magnetic wave propagation described using the model (1), (3) (e.g., $X$-rays and lasers) the frequency $\omega$ is so great that only phaseless values of $\left|\psi^{+}\right|$and $|f|$ can be measured in practice by modern technical devices; see, e.g., [13] and references therein.

Let

$$
\mathcal{M}_{\Lambda}=\cup_{E \in \Lambda} \mathcal{M}_{E}, \quad \text { where } \Lambda \subset \mathbb{R}_{+}=(0,+\infty) .
$$

We consider, in particular, the following inverse scattering problems for equation (1):

Problem 1.1. Reconstruct potential $v$ on $\mathbb{R}^{d}$ from its scattering amplitude $f$ on some appropriate $\mathcal{M}^{\prime} \subseteq \mathcal{M}_{\mathbb{R}_{+}}$.

Problem 1.2. Reconstruct potential $v$ on $\mathbb{R}^{d}$ from its phaseless scattering data $|f|^{2}$ on some appropriate $\mathcal{M}^{\prime} \subseteq \mathcal{M}_{\mathbb{R}_{+}}$.

Let $\mathcal{F}$ denote the Fourier transform defined by the formula

$$
\hat{\nu}(p)=\mathcal{F} \nu(p)=(2 \pi)^{-d} \int_{\mathbb{R}^{d}} e^{i p x} \nu(x) d x, p \in \mathbb{R}^{d},
$$

where $\nu$ is a test function.

In particular, for Problem 1.1, for $d \geq 2$, it is well known that the scattering amplitude $f$ at high energies uniquely determines $v$ via the formula

$$
\hat{v}(p)=f(k, l)+O\left(E^{-1 / 2}\right) \text { as } E \rightarrow+\infty, \quad(k, l) \in \mathcal{M}_{E}, \quad k-l=p \in \mathbb{R}^{d} ;
$$

see, for example, [10], [22]. For many other important results on Problem 1.1, see, for example, [7], [9], [26] and references therein.

On the other hand, for Problem 1.2 it is well known that the phaseless scattering data $|f|^{2}$ on $\mathcal{M}_{\mathbb{R}_{+}}$do not determine $v$ uniquely, in general; see, for example, [23].

In view of the aforementioned nonuniqueness for Problem 1.2 we also consider the Problem 1.3 formulated below.

Let $f$ be the initial scattering amplitude for $v$ satisfying (2) and $f_{j}$ be the scattering amplitude for

$$
v_{j}=v+w_{j}, \quad j=1, \ldots, n,
$$

where $w_{1}, \ldots, w_{n}$ are additional a priori known background scatterers such that

$$
\begin{aligned}
& w_{j} \in L^{\infty}\left(\mathbb{R}^{d}\right), \quad w_{j} \neq 0 \text { in } L^{\infty}\left(\mathbb{R}^{d}\right), \quad \text { supp } w_{j} \subset \Omega_{j}, \\
& \Omega_{j} \text { is an open bounded domain in } \mathbb{R}^{d}, \Omega_{j} \cap D=\varnothing, \\
& w_{j_{1}} \neq w_{j_{2}} \text { for } j_{1} \neq j_{2} \text { in } L^{\infty}\left(\mathbb{R}^{d}\right), \\
& j, j_{1}, j_{2} \in\{1, \ldots, n\} .
\end{aligned}
$$


Problem 1.3. Reconstruct potential $v$ on $\mathbb{R}^{d}$ from the phaseless scattering data $|f|^{2}$, $\left|f_{1}\right|^{2}, \ldots,\left|f_{n}\right|^{2}$ on some appropriate $\mathcal{M}^{\prime} \subseteq \mathcal{M}_{\mathbb{R}_{+}}$, for some appropriate background scatterers $w_{1}, \ldots, w_{n}$.

Problem 1.3 in dimension $d=1$ was considered in [1] for $n=1$. Problem 1.3 in dimension $d \geq 2$ was considered in [2], [3], [23], [24].

In particular, for Problem 1.3, for $d \geq 2, n=2$, analogs of formula (9) and related global uniqueness results were given in [23], [24]. Reconstruction results of [23], [24] on Problem 1.3, for $d \geq 2, n=2$, were strongly developed in [2], [3]. In particular, for the phaseless case with background scatterers, results of [2] include an analog of the algorithm of [22]. Related numerical implementation is also given in [2].

In the previous works [2], [3], [22], [23], [24] for uniqueness and efficiency of reconstruction in Problem 1.3, for $d \geq 2$, the phaseless scattering data $|f|^{2},\left|f_{1}\right|^{2},\left|f_{2}\right|^{2}$ at high energies and background $w_{1}, w_{2}$ were necessary. In the present work we show that these data can be reduced considerably. In particular, we show that already $\left|f_{1}\right|^{2}$ at high energies and $w_{1}$ uniquely determine $v$ via explicit formulas, under the condition that supp $v$ and supp $w_{1}$ are sufficiently disjoint ! If the latter condition is relaxed, we give similar formulas for finding $v$ from $|f|^{2},\left|f_{1}\right|^{2}$ at high energies and $w_{1}$. These formulas and related global uniqueness results are given in detail in Sections 3-6; see Theorems 4.1, 4.2, Corollaries 4.1, 4.2, Propositions 4.1, 4.2, Theorems 5.1, 5.2 and Theorems 6.1, 6.2. Note that our aforementioned reconstruction formulas include approximate reconstructions at fixed $E$ and related error estimates; see Sections 4.2, 5, 6.

Remark 1.1. The aforementioned results on Problem 1.3 consisting in finding $v$ from $\left|f_{1}\right|^{2}$ and $w_{1}$ can be also considered as results on Problem 1.2 with $v$ supported in $D \cup \Omega_{1}$, where $v$ is unknown in $D$ and $v=w_{1}$ in $\Omega_{1}$.

In addition, the present work involves considerations of the following problem of reconstruction from phaseless Fourier transforms, under assumptions (2), (9)-(11) (with $L^{1}$ in place of $L^{\infty}$ for more generality).

Problem 1.4. Reconstruct $v$ from the phaseless Fourier transforms $|\mathcal{F} v|^{2},\left|\mathcal{F} v_{1}\right|^{2}, \ldots,\left|\mathcal{F} v_{n}\right|^{2}$ and background $w_{1}, \ldots, w_{n}$.

Problem 1.4 can be considered as Problem 1.3 in the Born approximation at high energies or/and for small $v, w_{1}, \ldots, w_{n}$; see formulas (16), (18).

In the literature the problem of finding $v$ from $|\mathcal{F} v|^{2}$ is known as the phase retrieval problem; see [20], [4], [8] and references therein. The problem of finding $v$ from $\left|\mathcal{F} v_{1}\right|^{2}$ and $w_{1}$ was considered, in particular, in [28], [21]. Problem 1.4 in the framework of solving Problem 1.3 was considered in [3], [2], [23], [24] and we continue such considerations in the present work.

The results of the present work on Problem 1.4 are given in Section 3 and consist in Theorems 3.1 and 3.2. Actually, Theorems 3.1 is a proper mathematical formalization of some of considerations of [21] related with finding $v$ from $\left|\mathcal{F} v_{1}\right|^{2}$ and $w_{1}$, under the condition that supp $v$ and supp $w_{1}$ are sufficiently disjoint.

In addition to Problems 1.2, 1.3, 1.4, there are also other possible formulations of phaseless inverse scattering problems for equation (1) and for other equations of wave propagation. In connection with such formulations and related results, see, for example, [12]-[14], [17]-[19], [23], [25]-[27], [29]-[31] and references therein.

Finally, note that the further structure of the present article is as follows. In Section 2 we recall some known results on direct scattering for equation (1) under assumptions (2) (or when $v$ is replaced by $v_{j}$ of $(10),(11)$ ). The results of the present work on Problem 1.4 are given in Section 3. The results of the present work on Problem 1.3 (and on Problem 1.2, see Remark 1.1) are given in Sections 4, 5, 6. In addition, estimates of Sections 5 and 6 are proved in Sections 7 and 8. 


\section{Preliminares on direct scattering for equation (1)}

For equation (1), under assumptions (2), we consider the scattering solutions $\psi^{+}$specified by (4) and the scattering amplitude $f$ arising in (4), (6). We recall that $\psi^{+}$satisfy the LippmannSchwinger integral equation

$$
\begin{aligned}
& \psi^{+}(x, k)=e^{i k x}+\int_{D} G^{+}(x-y, k) v(y) \psi^{+}(y, k) d y, \\
& G^{+}(x, k)=-(2 \pi)^{-d} \int_{\mathbb{R}^{d}} \frac{e^{i \xi x} d \xi}{\xi^{2}-k^{2}-i 0}=G_{0}^{+}(|x|,|k|, d),
\end{aligned}
$$

where $x, k \in \mathbb{R}^{d}, k^{2}=E$, and for $f$ the following formula holds:

$$
f(k, l)=(2 \pi)^{-d} \int_{D} e^{-i l y} v(y) \psi^{+}(y, k) d y
$$

where $k, l \in \mathbb{R}^{d}, k^{2}=l^{2}=E>0$; see, for example, [5], [26] and references therein.

We also recall that for any $s>1 / 2$, the following Agmon estimate holds:

$$
\left\|<x>^{-s} G^{+}(k)<x>^{-s}\right\|_{L^{2}\left(\mathbb{R}^{d}\right) \rightarrow L^{2}\left(\mathbb{R}^{d}\right)}=a_{0}(d, s)|k|^{-1},|k| \geq 1,
$$

where $\langle x\rangle$ denotes the multiplication operator by the function $\left(1+|x|^{2}\right)^{1 / 2}, G^{+}(k)$ denotes the integral operator such that

$$
G^{+}(k) u(x)=\int_{\mathbb{R}^{d}} G^{+}(x-y, k) u(y) d y,
$$

where $G^{+}(x, k)$ is defined in (12), $u$ is a test function. See, for example, the proof of (14) given in [9]; following this proof an explicit estimate for $a_{0}(d, s)$ can be given.

Using (12), (13), (14) one can show that, under assumptions (2), (10), (11), formula (9) is valid and the following formulas hold:

$$
\left|\mathcal{F} v_{j}(p)\right|^{2}=\left|f_{j}(k, l)\right|^{2}+O\left(E^{-1 / 2}\right) \text { as } E \rightarrow+\infty,(k, l) \in \mathcal{M}_{E}, k-l=p \in \mathbb{R}^{d},
$$

where $f_{j}$ is the scattering amplitude for $v_{j}, j=0, \ldots, n$, and $v_{0}=v, f_{0}=f, v_{j}=v+w_{j}, j \geq 1$; see, for example, [22], [24] and references therein.

We recall that formulas (9), (16) hold for any fixed $p \in \mathbb{R}^{d}$ for $d \geq 2$.

Suppose that, in addition to (2), (10), (11), we have that

$$
\max \left(\|v\|_{\infty},\left\|w_{j}\right\|_{\infty}\right) \leq \eta
$$

where $j=1, \ldots, n,\|\cdot\|_{\infty}=\|\cdot\|_{L^{\infty}\left(\mathbb{R}^{d}\right)}$. Then the following more precise version of (16) holds (see formula (2.15) in [24]):

$$
\begin{aligned}
& \left.|| \mathcal{F} v_{j}(p)\right|^{2}-\left|f_{j}(k, l)\right|^{2} \mid \leq c\left(D \cup \Omega_{j}\right) \eta^{3} E^{-1 / 2},(k, l) \in \mathcal{M}_{E}, k-l=p \\
& \text { for } E^{1 / 2} \geq \rho\left(D \cup \Omega_{j}, \eta\right),
\end{aligned}
$$

where $j=0, \ldots, n, \Omega_{0}=\varnothing$, and $c>0, \rho \geq 1$ are the constants defined by the formulas:

$$
\begin{aligned}
& \rho(\mathcal{U}, \eta)=\max \left(2 a_{0}(d, \sigma / 2) c_{2}(\mathcal{U}, \sigma) \eta, 1\right), \\
& c(\mathcal{U})=6(2 \pi)^{-2 d} a_{0}(d, \sigma / 2)\left(c_{1}(d, \sigma)\right)^{4}\left(c_{2}(d, \sigma)\right)^{3}, \\
& c_{1}(d, \sigma)=\left(\int_{\mathbb{R}^{d}} \frac{d x}{\left(1+|x|^{2}\right)^{\sigma / 2}}\right)^{1 / 2}, \\
& c_{2}(\mathcal{U}, \sigma)=\sup _{x \in \mathcal{U}}\left(1+|x|^{2}\right)^{\sigma / 2},
\end{aligned}
$$

for some fixed $\sigma>d, a_{0}(d, \sigma)$ is the constant of (14), $\mathcal{U}$ is open bounded domain in $\mathbb{R}^{d}$.

Remark 2.1. The constants $c, \rho$ have the following monotonicity properties:

$$
\begin{aligned}
& c\left(\mathcal{U}_{1} \cup \mathcal{U}_{2}\right) \geq c\left(\mathcal{U}_{1}\right), \\
& \rho\left(\mathcal{U}_{1} \cup \mathcal{U}_{2}, \eta\right) \geq \rho\left(\mathcal{U}_{1}, \eta\right), \quad \rho\left(\mathcal{U}, \eta_{2}\right) \geq \rho\left(\mathcal{U}, \eta_{1}\right) \text { for } \eta_{2}>\eta_{1},
\end{aligned}
$$

where $\mathcal{U}, \mathcal{U}_{1}, \mathcal{U}_{2}$ are bounded domains in $\mathbb{R}^{d}, \eta, \eta_{1}, \eta_{2}>0$. 


\section{Reconstruction from phaseless Fourier transforms}

We consider $v$ and $w$ such that

$$
\begin{aligned}
& v, w \in L_{1, l o c}\left(\mathbb{R}^{d}\right), w \neq 0, d \geq 1, \\
& \operatorname{supp} v \subset D, \text { supp } w \subset \Omega, \\
& D, \Omega \text { are open convex and bounded domains in } \mathbb{R}^{d} .
\end{aligned}
$$

Let

$$
\begin{aligned}
& \operatorname{diam} \mathcal{U}=\sup _{x, y \in \mathcal{U}}|x-y| \\
& \mathcal{U}_{1}+\mathcal{U}_{2}:=\left\{x+y: x \in \mathcal{U}_{1}, y \in \mathcal{U}_{2}\right\}
\end{aligned}
$$

where $\mathcal{U}, \mathcal{U}_{1}, \mathcal{U}_{2}$ are bounded sets in $\mathbb{R}^{d}$.

Note that

$$
\begin{aligned}
& B_{r_{1}}\left(a_{1}\right)+B_{r_{2}}\left(a_{2}\right)=B_{r_{1}+r_{2}}\left(a_{1}+a_{2}\right), \text { where } \\
& B_{r}(a)=\left\{x \in \mathbb{R}^{d}:|x-a|<r\right\}, a \in \mathbb{R}^{d}, r>0 .
\end{aligned}
$$

We suppose that the Fourier transformation $\mathcal{F}$ is defined by formula (8).

In this case $\mathcal{F}^{-1}$ is given by the formula

$$
\mathcal{F}^{-1} \varphi(x):=\int_{\mathbb{R}^{d}} e^{-i p x} \varphi(p) d p,
$$

where $\varphi$ is a test function.

Theorem 3.1. Let $v$, $w$ satisfy (25)-(27), where $\operatorname{dist}(D, \Omega)>\operatorname{diam} D$. Then $|\mathcal{F}(v+w)|^{2}$ and $w$ uniquely determine $v$ by the formulas

$$
\begin{aligned}
& \mathcal{F} v(p):=(\overline{\mathcal{F} w}(p))^{-1} \mathcal{F} q(p), p \in \mathbb{R}^{d}, \\
& q(x):=\chi_{D-\Omega}(x)\left(u(x)-(2 \pi)^{-d} \int_{y \in \Omega} w(x+y) \overline{w(y)} d y\right), \\
& u(x):=\mathcal{F}^{-1}\left(|\mathcal{F}(v+w)|^{2}\right)(x),
\end{aligned}
$$

where $\chi_{D-\Omega}$ is the characteristic function of the set $D-\Omega$.

As it is was already mentioned in Introduction, Theorem 3.1 can be considered as a proper mathematical formalization of some of considerations of [21] related with finding $v$ from $|\mathcal{F}(v+w)|^{2}$ and $w$, under the condition that supp $v$ and supp $w$ are sufficiently disjoint.

Theorem 3.2. Let $v$, $w$ satisfy (25)-(27), where $\operatorname{dist}(D, \Omega)>0$. Then $|\mathcal{F}(v)|^{2},|\mathcal{F}(v+w)|^{2}$ and $w$ uniquely determine $v$ by the formulas

$$
\begin{aligned}
& \mathcal{F} v(p):=(\overline{\mathcal{F} w}(p))^{-1} \mathcal{F} q(p), p \in \mathbb{R}^{d}, \\
& q(x):=\chi_{D-\Omega}(x)\left(u(x)-(2 \pi)^{-d} \int_{y \in \Omega} w(x+y) \overline{w(y)} d y\right), \\
& u(x):=\mathcal{F}^{-1}\left(|\mathcal{F}(v+w)|^{2}\right)(x)-\mathcal{F}^{-1}\left(|\mathcal{F} v|^{2}\right)(x) .
\end{aligned}
$$

Under our assumptions on $w$, we have that

$$
\mu\left(\left\{p \in \mathbb{R}^{d}: \mathcal{F} w(p)=0\right\}\right)=0,
$$

where $\mu(\mathcal{A})$ denotes the $\mathrm{d}$-dimentional Lebesgue measure of a set $\mathcal{A}$. Therefore, formulas (32), (35) are correctly defined inspite of the factor $(\overline{\mathcal{F} w}(p))^{-1}$. 
In order to prove Theorems 3.1 and 3.2 we use, in particular, that

$$
\begin{aligned}
& \mathcal{F}^{-1}\left(\varphi_{1} \varphi_{2}\right)=(2 \pi)^{-d}\left(\mathcal{F}^{-1} \varphi_{1}\right) *\left(\mathcal{F}^{-1} \varphi_{2}\right), \\
& \left(\nu_{1} * \nu_{2}\right)(x):=\int_{\mathbb{R}^{d}} \nu_{1}(x-y) \nu_{2}(y) d y \\
& \overline{\mathcal{F} \nu}=\mathcal{F} \widetilde{\nu}, \quad \widetilde{\nu}(x)=\overline{\nu(-x)} \\
& (2 \pi)^{-d} \mathcal{F}\left(\nu_{1} * \nu_{2}\right)=\mathcal{F} \nu_{1} \mathcal{F} \nu_{2}
\end{aligned}
$$

where $\varphi_{1}, \varphi_{2}, \nu, \nu_{1}, \nu_{2}$ are test functions.

Proof of Theorem 3.1. Using formulas (39), (40), (41) for $\varphi_{1}=\mathcal{F}(v+w), \varphi_{2}=\overline{\mathcal{F}(v+w)}, \nu=$ $\nu_{1}=v+w, \nu_{2}=\widetilde{v}+\widetilde{w}$, we obtain that

$$
\begin{aligned}
I & =(2 \pi)^{d} \mathcal{F}^{-1}\left(|\mathcal{F}(v+w)|^{2}\right)=(v+w) *(\widetilde{v}+\widetilde{w})= \\
& =\int_{\mathbb{R}^{d}}(v(x-y)+w(x-y) \overline{(v(-y)+w(-y))} d y= \\
& =\int_{\mathbb{R}^{d}} v(x-y) \overline{v(-y)} d y+\int_{\mathbb{R}^{d}} w(x-y) \overline{v(-y)} d y+\int_{\mathbb{R}^{d}} v(x-y) \overline{w(-y)} d y+ \\
& +\int_{\mathbb{R}^{d}} w(x-y) \overline{w(-y)} d y=: I_{1}+I_{2}+I_{3}+I_{4} .
\end{aligned}
$$

Let

$$
B_{r}=\left\{x \in \mathbb{R}^{d}:|x|<r\right\} .
$$

Note that

$$
\begin{aligned}
& I_{1}(x)=\int_{y \in-D} v(x-y) \overline{v(-y)} d y, \\
& \text { supp } I_{1} \subset B_{\text {diam } D}, \\
& I_{2}(x)=\int_{y \in-D} w(x-y) \overline{v(-y)} d y, \\
& \operatorname{supp} I_{2} \subset \Omega-D, \\
& I_{3}(x)=\int_{-y \in \Omega} v(x-y) \overline{w(-y)} d y, \\
& \text { supp } I_{3} \subset D-\Omega, \\
& I_{4}(x)=\int_{-y \in \Omega} w(x-y) \overline{w(-y)} d y, \\
& \text { supp } I_{4}(x) \subset B_{\text {diam } \Omega},
\end{aligned}
$$

where $\Omega-D, D-\Omega, B_{r}$ are defined according to (29), (44).

Property (46) follows from the observation that if $y \in D, x+y \in D$, then $x \in B_{\text {diam } D}$; property (48) follows from the observation that if $y \in D, x+y \in \Omega$, then $x \in \Omega-D$; and the derivation of (50), (52) is similar.

Using (27) and the assumption that $\operatorname{dist}(D, \Omega)>\operatorname{diam} D$ one can see that

$$
\operatorname{dist}(D, \Omega)>\operatorname{diam} D \Leftrightarrow \forall x \in D, y \in \Omega:|x-y|>\operatorname{diam} D \Leftrightarrow B_{\operatorname{diam} D} \cap(D-\Omega)=\varnothing .
$$

Since $D$ and $\Omega$ are convex, the sets $D-\Omega$ and $\Omega-D$ are also convex and reciprocally symmetric. Therefore, if their intersection is nonempty, it contains the point 0. But formula (53) implies that $0 \notin D-\Omega$. Thus, we have that

$$
(D-\Omega) \cap(\Omega-D)=\varnothing .
$$


From (53), (54) we conclude that

$$
\begin{aligned}
& (D-\Omega) \cap\left(B_{\text {diam } D} \cup(\Omega-D)\right)=\varnothing, \\
& (\Omega-D) \cap\left(B_{\text {diam } D} \cup(D-\Omega)\right)=\varnothing .
\end{aligned}
$$

Formulas (43), (46), (48), (50), (55), (56) imply that

$$
\begin{aligned}
& I_{2}(x)=\chi_{\Omega-D}(x)\left(I(x)-I_{4}(x)\right), \\
& I_{3}(x)=\chi_{D-\Omega}(x)\left(I(x)-I_{4}(x)\right) .
\end{aligned}
$$

Using (42), (47), (49) we have that

$$
\begin{aligned}
& (2 \pi)^{-d} \mathcal{F} I_{2}=\mathcal{F} \widetilde{v} \mathcal{F} w, \\
& (2 \pi)^{-d} \mathcal{F} I_{3}=\mathcal{F} v \mathcal{F} \widetilde{w} .
\end{aligned}
$$

The formulas (32)-(34) follow from (41), (43), (58), (60). This completes the proof of Theorem 3.1.

Proof of Theorem 3.2. Formulas (43)-(52), (59), (60) remain valid under the assumptions of Theorem 3.2.

The assumption $\operatorname{dist}(D, \Omega)>0$ implies that

$$
0 \notin D-\Omega
$$

Using formula (61) and the proof of formula (54) we obtain that formula (54) also remains valid under the assumptions of Theorem 3.2.

Formulas (43), (48), (50) and (54) imply that

$$
\begin{aligned}
& I_{2}(x)=\chi_{\Omega-D}(x)\left(I(x)-I_{1}(x)-I_{4}(x)\right), \\
& I_{3}(x)=\chi_{D-\Omega}(x)\left(I(x)-I_{1}(x)-I_{4}(x)\right) .
\end{aligned}
$$

The formulas (35)-(37) follow from (43), (60), (63). This completes the proof of Theorem 3.2.

Remark 3.1. Under the assumptions of Theorem 3.1, there exist $\varepsilon>0$, an open $\varepsilon$-neighbourhood $\mathcal{N}_{\varepsilon}(D-\Omega)$ of $D-\Omega$, and a function $\chi_{D-\Omega, \varepsilon} \in C^{\infty}\left(\mathbb{R}^{d}\right)$, such that

$$
\begin{aligned}
& \chi_{D-\Omega, \varepsilon}(x)=1, x \in D-\Omega, \\
& \chi_{D-\Omega, \varepsilon}(x)=0, x \in \mathbb{R}^{d} \backslash \mathcal{N}_{\varepsilon}(D-\Omega), \\
& (\Omega-D) \cap \mathcal{N}_{\varepsilon}(D-\Omega)=\varnothing, \\
& B_{\text {diam } D} \cap \mathcal{N}_{\varepsilon}(D-\Omega)=\varnothing .
\end{aligned}
$$

In addition, formulas (32)-(34) remain valid with $\chi_{D-\Omega}$ replaced by $\chi_{D-\Omega, \varepsilon}$.

Remark 3.2. Under the assumptions of Theorem 3.2, there exist $\varepsilon>0$, an open $\varepsilon$-neighbourhood $\mathcal{N}_{\varepsilon}(D-\Omega)$ of $D-\Omega$, and a function $\chi_{D-\Omega, \varepsilon} \in C^{\infty}\left(\mathbb{R}^{d}\right)$, such that properties (64)-(66) are fulfilled. In addition, formulas (35)-(37) remain valid with $\chi_{D-\Omega}$ replaced by $\chi_{D-\Omega, \varepsilon}$.

Note that Remarks 3.1 and 3.2 are used in estimates of Sections 5 and 6 .

\section{Reconstruction formulas and uniqueness results for Problem 1.3}

In this section we give reconstruction formulas and global uniqueness results for Problem 1.3, for $d \geq 2, n=1$, and for Problem 1.2, for $d \geq 2$ (see Remark 1.1). These reconstruction formulas and global uniqueness results develop considerably related studies of [23], [24] on Problem 1.3, for $d \geq 2, n=2$ and $n=1$. 


\subsection{Uniqueness results}

Theorem 4.1. Let $v, w_{1}$ satisfy (2), (11), where $D, \Omega=\Omega_{1}$ satisfy (27), $\operatorname{dist}\left(D, \Omega_{1}\right)>\operatorname{diam} D$, and $d \geq 2$. Then $\left|f_{1}\right|^{2}$ and $w_{1}$ uniquely determine $v$ via formulas (32)-(34), where $w=w_{1}$, and formula (16) for $j=1$.

Theorem 4.2. Let $v, w_{1}$ satisfy (2), (11), where $D, \Omega=\Omega_{1}$ satisfy (27), dist $\left(D, \Omega_{1}\right)>0$, and $d \geq 2$. Then $|f|^{2},\left|f_{1}\right|^{2}$ and $w_{1}$ uniquely determine $v$ via formulas (35)-(37), where $w=w_{1}$, and formula (16) for $j=0,1$.

Theorems 4.1 and 4.2 follow directly from formula (16) and Theorems 3.1 and 3.2.

In addition to $f, f_{1}$ on $\mathcal{M}_{E}$, we also consider $\left.f\right|_{\Gamma_{E}},\left.f_{1}\right|_{\Gamma_{E}}$, where

$$
\begin{aligned}
& \Gamma_{E}=\left\{k=k_{E}(p), l=l_{E}(p): p \in \bar{B}_{2 \sqrt{E}}\right\}, \\
& k_{E}(p)=p / 2+\left(E-p^{2} / 4\right)^{1 / 2} \gamma(p), l_{E}(p)=-p / 2+\left(E-p^{2} / 4\right)^{1 / 2} \gamma(p), \\
& B_{r}=\left\{p \in \mathbb{R}^{d}:|p|<r\right\}, \bar{B}_{r}=\left\{r \in \mathbb{R}^{d}:|p| \leq r\right\}, r>0,
\end{aligned}
$$

where $\gamma$ is a piecewise continuous vector-function on $\mathbb{R}^{d}, d \geq 2$, such that

$$
|\gamma(p)|=1, \gamma(p) p=0, p \in \mathbb{R}^{d} .
$$

We recall that

$$
\Gamma_{E} \subset \mathcal{M}_{E}, \operatorname{dim} \Gamma_{E}=d, \operatorname{dim} \mathcal{M}_{E}=2 d-2, E>0, d \geq 2
$$

We also consider

$$
\mathcal{M}_{\Lambda}=\cup_{E \in \Lambda} \mathcal{M}_{E}, \Gamma_{\Lambda}=\cup_{E \in \Lambda} \Gamma_{E}
$$

where $\Lambda \subseteq \mathbb{R}_{+}=(0,+\infty)$.

Let, for example, $\Lambda$ be of the following form

$$
\Lambda=\left\{E_{j} \in \mathbb{R}_{+}: j \in \mathbb{N}, E_{j} \rightarrow \infty, \text { as } j \rightarrow \infty\right\} .
$$

Theorems 4.1 and 4.2 , where we use formula (16) with $k=k_{E_{j}}(p), l=l_{E_{j}}(p)$, imply the following corollaries:

Corollary 4.1. Let the assumptions of Theorem 4.1 hold. Let $\Lambda$ be of the form (73). Then $\left|f_{1}\right|^{2}$ on $\Gamma_{\Lambda}$ and background $w_{1}$ uniquely determine $v$ in $L^{\infty}\left(\mathbb{R}^{d}\right)$.

Corollary 4.2. Let the assumptions of Theorem 4.2 hold. Let $\Lambda$ be of the form (73). Then $S=\left\{|f|^{2},\left|f_{1}\right|^{2}\right\}$ on $\Gamma_{\Lambda}$ and background $w_{1}$ uniquely determine $v$ in $L^{\infty}\left(\mathbb{R}^{d}\right)$.

We also consider $\Lambda$ of the form

$$
\Lambda=\left\{E_{j} \in \mathbb{R}_{+}: j \in \mathbb{N}, E_{j_{1}} \neq E_{j_{2}} \text { for } j_{1} \neq j_{2}, E_{j} \rightarrow E_{*} \text {, as } j \rightarrow \infty\right\}, E_{*}>0 .
$$

Proceeding from Theorems 4.1 and 4.2 we obtain the following results:

Proposition 4.1. Let the assumptions of Theorem 4.1 hold, and $v, w_{1}$ be real-valued. Let $\Lambda$ be of the form (74). Then $\left|f_{1}\right|^{2}$ on $\Gamma_{\Lambda}$ and background $w_{1}$ uniquely determine $v$.

Proposition 4.2. Let the assumptions of Theorem 4.2 hold, and $v, w_{1}$ be real-valued. Let $\Lambda$ be of the form (74). Then $S=\left\{|f|^{2},\left|f_{1}\right|^{2}\right\}$ on $\Gamma_{\Lambda}$ and background $w_{1}$ uniquely determine $v$. 
The proof of Propositions 4.1, 4.2 is similar to the proof of Theorem 2.2 of [24].

Remark 4.1 Corollary 4.2 and Proposition 4.2 of the present work develop the result of Proposition 2.2 of [24], where it was show that:

(A) There are not more then two different complex-valued potentials $v$ satisfying (2) with given $S=\left\{|f|^{2},\left|f_{1}\right|^{2}\right\}$ on $\Gamma_{\Lambda}$ and background complex-valued potential $w_{1}$ satisfying $(11), w_{1} \neq 0$ in $L^{\infty}\left(\mathbb{R}^{d}\right)$, where $\Lambda$ is defined as in (73);

(B) There are not more than two different real-valued potentials $v$ satisfying (2) with given $S=\left\{|f|^{2},\left|f_{1}\right|^{2}\right\}$ on $\Gamma_{\Lambda}$ and background real-valued potential $w_{1}$ satisfying $(11), w_{1} \neq 0$ in $L^{\infty}\left(\mathbb{R}^{d}\right)$, where $\Lambda$ is defined as in (74).

In addition, the reconstruction of Corollary 4.2 (based on formulas mentioned in Theorem 4.2) of the present work does not involve an analytic continuation in contrast with item (A) of Proposition 2.2 of [24].

\subsection{Approximate reconstruction formulas}

Suppose that the assumptions of Theorem 4.1 are valid. Then, at fixed $E$, proceeding from Theorem 4.1 and Remark 3.1 we reconstruct $v(x)$ as $v_{a p p r}(x, E)$, where

$$
\begin{aligned}
& v_{\text {appr }}(x, E):=\left(\mathcal{F}^{-1} \hat{v}_{\text {appr }}\right)(x, E), x \in D, \\
& \hat{v}_{\text {appr }}(p, E):=\left\{\begin{array}{c}
\left(\overline{\mathcal{F} w_{1}(p)}\right)^{-1}\left(\mathcal{F} q_{\text {appr }}\right)(p) \text { for } p \in B_{(2-\delta) \sqrt{E}}, \\
0 \text { for } p \in \mathbb{R}^{d} \backslash B_{(2-\delta) \sqrt{E}},
\end{array}\right. \\
& q_{\text {appr }}(x, E):=\chi_{D-\Omega, \varepsilon}(x)\left(u_{\text {appr }}(x, E)-(2 \pi)^{-d} \int_{y \in \Omega} w_{1}(x+y) \overline{w_{1}(y)} d y\right), \\
& u_{\text {appr }}(x, E):=\left(\mathcal{F}^{-1} h\right)(x), \\
& h(p, E):=\left\{\begin{array}{l}
\left|f_{1}\left(k_{E}(p), l_{E}(p)\right)\right|^{2} \text { for } p \in B_{2 \sqrt{E}}, \\
\left|\mathcal{F} w_{1}(p, E)\right|^{2} \text { for } p \in \mathbb{R}^{d} \backslash B_{2 \sqrt{E}},
\end{array}\right.
\end{aligned}
$$

where $k_{E}(p), l_{E}(p)$ are defined in $(68), \chi_{D-\Omega, \varepsilon}$ is the function of Remark 3.1, and $\delta \in(0,2)$.

Suppose that the assumptions of Theorem 4.2 are valid. Then, at fixed $E$, proceeding from Theorem 4.2 and Remark 3.2 we construct $v(x)$ as $v_{\text {appr }}(x, E)$, where $v_{\text {appr }}(x, E)$ is given by formulas (75)-(78) with

$$
h(p, E):=\left\{\begin{array}{l}
\left|f_{1}\left(k_{E}(p), l_{E}(p)\right)\right|^{2}-\left|f\left(k_{E}(p), l_{E}(p)\right)\right|^{2} \text { for } p \in B_{2 \sqrt{E}}, \\
\left|\mathcal{F} w_{1}(p, E)\right|^{2} \text { for } p \in \mathbb{R}^{d} \backslash B_{2 \sqrt{E}} .
\end{array}\right.
$$

In Sections 5 and 6 we give error estimates for $\hat{v}_{\text {appr }}$ and $v_{\text {appr }}$. These error estimates develop considerably related studies of [23], [24], and [3] on Problem 1.3, for $d \geq 2, n \geq 2$.

\section{$5 \quad$ Error estimates for $\hat{v}_{a p p r}$}

In this section we estimate $\left|\hat{v}(p)-\hat{v}_{\text {appr }}(p, E)\right|$ for $p \in B_{(2-\delta) \sqrt{E}}$, where $\hat{v}_{\text {appr }}$ is defined in Subsection $4.2, \hat{v}=\mathcal{F} v$.

Note that the following estimate holds:

$$
\left|\mathcal{F} \chi_{D-\Omega, \varepsilon}(p)\right| \leq \frac{C_{1}(\sigma)}{(1+|p|)^{\sigma}}, p \in \mathbb{R}^{d}, \sigma \geq 0
$$

where $\chi_{D-\Omega, \varepsilon}$ is the function of Remarks 3.1, 3.2, $C_{1}(\sigma)=C_{1}\left(\sigma, \chi_{D-\Omega, \varepsilon}\right)$ is a positive constant.

Let

$$
C_{2}(\sigma)=\int_{\mathbb{R}^{d}} \frac{d p}{(1+|p|)^{\sigma}}, \sigma>d
$$

Let $\mu(\mathcal{U})$ denote the Lebesgue measure of a bounded domain $\mathcal{U} \subset \mathbb{R}^{d}$. 
Theorem 5.1. Suppose that the assumptions of Theorem 4.1 are valid, and $v, w_{1}$ satisfy (17). Let $\hat{v}_{\text {appr }}$ be defined via (76)-(79). Then:

$$
\begin{aligned}
& \left|\hat{v}(p)-\hat{v}_{a p p r}(p, E)\right| \leq\left|\hat{w}_{1}(p)\right|^{-1}\left(C_{3} E^{-1 / 2} \eta^{3}+\frac{C_{4} \eta^{2}}{\left(1+\delta E^{1 / 2}\right)^{\sigma-d-\alpha}}\right) \\
& \text { for } p \in B_{(2-\delta) \sqrt{E}}, E^{1 / 2} \geq \rho(D \cup \Omega, \eta), \\
& C_{3}=c(D \cup \Omega) C_{1}\left(\sigma_{1}\right) C_{2}\left(\sigma_{1}\right), \sigma_{1}>d, \\
& C_{4}=(2 \pi)^{-2 d}\left(\mu(D)^{2}+2 \mu(D) \mu(\Omega)\right) C_{1}(\sigma) C_{2}(d+\alpha), \alpha>0, \sigma-d-\alpha>0,
\end{aligned}
$$

where $c, \rho$ are the constants of $(18), C_{1}, C_{2}$ are the constants of $(81),(82), \delta \in(0,2)$ is fixed.

Theorem 5.2. Suppose that the assumptions of Theorem 4.2 are valid, and $v, w_{1}$ satisfy (17). Let $\hat{v}_{\text {appr }}$ be defined via (76)-(78), (80). Then:

$$
\begin{aligned}
& \left|\hat{v}(p)-\hat{v}_{a p p r}(p, E)\right| \leq\left|\hat{w}_{1}(p)\right|^{-1}\left(C_{5} E^{-1 / 2} \eta^{3}+\frac{C_{6} \eta^{2}}{\left(1+\delta E^{1 / 2}\right)^{\sigma-d-\alpha}}\right) \\
& \text { for } p \in B_{(2-\delta) \sqrt{E}}, E^{1 / 2} \geq \rho(D \cup \Omega, \eta), \\
& C_{5}=(c(D)+c(D \cup \Omega)) C_{1}\left(\sigma_{1}\right) C_{2}\left(\sigma_{1}\right), \sigma_{1}>d, \\
& C_{6}=2(2 \pi)^{-2 d} \mu(D) \mu(\Omega) C_{1}(\sigma) C_{2}(d+\alpha), \alpha>0, \sigma-d-\alpha>0,
\end{aligned}
$$

where $c, \rho$ are the constants of $(18), C_{1}, C_{2}$ are the constants of $(81),(82), \delta \in(0,2)$ is fixed.

The estimates of Theorems 5.1, 5.2 can be considered as error estimates for $\hat{v}_{a p p r}(p, E)$ at high energies $E$.

Theorems 5.1, 5.2 are proved in Section 7.

In addition, it may be of interest to consider estimates (83), (86) for small $v, w_{1}$, that is for small $\eta$. In this case it is convenient to suppose also that

$$
\left|\mathcal{F} w_{1}(p)\right| \geq \eta\left|\hat{w}_{0}(p)\right|, \forall p \in \mathbb{R}^{d},
$$

where $\hat{w}_{0}$ is independent of $\eta$, and

$$
\begin{aligned}
& \hat{w}_{0}(p)=\mathcal{F} w_{0}(p), \\
& w_{0} \in L^{\infty}\left(\mathbb{R}^{d}\right), \text { supp } w_{0} \subset \Omega=\Omega_{1}, w_{0} \neq 0 .
\end{aligned}
$$

Then estimate (83) takes the form

$$
\begin{aligned}
& \left|\hat{v}(p)-\hat{v}_{a p p r}(p, E)\right| \leq\left|\hat{w}_{0}(p)\right|^{-1}\left(C_{3} E^{-1 / 2} \eta^{2}+\frac{C_{4} \eta}{\left(1+\delta E^{1 / 2}\right)^{\sigma-d-\alpha}}\right) \\
& \text { for } p \in B_{(2-\delta) \sqrt{E}}, E^{1 / 2} \geq \rho(D \cup \Omega, \eta), \alpha>0, \sigma>d+\alpha,
\end{aligned}
$$

and estimate (86) takes the form

$$
\begin{aligned}
& \left|\hat{v}(p)-\hat{v}_{\text {appr }}(p, E)\right| \leq\left|\hat{w}_{0}(p)\right|^{-1}\left(C_{5} E^{-1 / 2} \eta^{2}+\frac{C_{6} \eta}{\left(1+\delta E^{1 / 2}\right)^{\sigma-d-\alpha}}\right) \\
& \text { for } p \in B_{(2-\delta) \sqrt{E}}, E^{1 / 2} \geq \rho(D \cup \Omega, \eta), \alpha>0, \sigma>d+\alpha .
\end{aligned}
$$

One can see that estimates (92), (93) are very efficient for small $\eta$ and large $E$, because in this case $E^{-1 / 2} \eta^{2}$ and $\eta\left(1+\delta E^{1 / 2}\right)^{-\sigma+d+\alpha}$ are very small.

Remark 5.1. The background $w_{1}$ can be chosen as real-valued non-negative continious compactly supported function on $\mathbb{R}^{d}$ such that

$$
\widehat{w_{1}}(p)=\widehat{\widehat{w}_{1}}(p) \geq c_{3}(1+|p|)^{-\beta}, p \in \mathbb{R}^{d},
$$

for $\beta>d$ and $c_{3}>0$; see, for example, Lemma 1 in [3]. Property (94) can be convenient in the framework of applications of the error estimates $(83),(86)$.

Remark 5.2. If $\widehat{w}_{1}(p)$ in (76), (83), (86) has zeros, then the definition of $\hat{v}_{a p p r}(p, E)$ can be modified in neighborhoods of these zeros in a similar way with interpolations of Section 4 of [3]. 


\section{$6 \quad$ Error estimates for $v_{\text {appr }}$}

In this section we estimate $\left|v(x)-v_{\text {appr }}(x, E)\right|$ for $x \in D$, where $v_{\text {appr }}$ is defined as in Subsection 4.2 with $\delta$ depending on $E$, and $v, w_{1}$ satisfy the assumptions of Theorem 5.1 or 5.2. In addition, for simplicity, we assume that $v \in W^{m, 1}\left(\mathbb{R}^{d}\right), m>d$, and $w_{1}$ satisfies (94), where

$$
\begin{aligned}
& W^{m, 1}\left(\mathbb{R}^{d}\right)=\left\{u: \partial^{J} u \in L^{1}\left(\mathbb{R}^{d}\right),|J| \leq m\right\}, \\
& \|u\|_{m, 1}=\max _{|J| \leq m}\left\|\partial^{J} u\right\|_{L^{1}\left(\mathbb{R}^{d}\right)}, m \in \mathbb{N} \cup 0 .
\end{aligned}
$$

Next, we assume that

$$
\begin{aligned}
& v_{\text {appr }}(x, E)=\int_{B_{r_{1}(E)}} e^{-i x p} \hat{v}_{\text {appr }}(p, E) d p, x \in D, \\
& r_{1}(E)=(2-\delta(E)) \sqrt{E}=2 \tau E^{\gamma}, \gamma=\frac{1}{2} \frac{1}{m+\beta},
\end{aligned}
$$

where $E \geq \rho^{2}(D \cup \Omega, \eta) \geq 1$ as in Theorems 5.1, 5.2, $\beta$ is the number of $(94), \tau \in(0,1)$ is fixed. One can see that $\delta(E) \in(0,2)$ under the assumptions of formula (96).

Note that if $v \in W^{m, 1}\left(\mathbb{R}^{d}\right), m \geq 0$, then the following estimate holds:

$$
|\hat{v}(p)| \leq \frac{C_{7}(m)}{(1+|p|)^{m}}, p \in \mathbb{R}^{d},
$$

where $C_{7}(m)=C_{7}\left(m, d,\|v\|_{m, 1}\right)$ is a positive constant.

Let

$$
\gamma_{1}=\frac{1}{2} \frac{m-d}{m+\beta}, \quad \gamma_{2}=\frac{\sigma-d-\alpha-1}{2},
$$

where $\alpha>0, \sigma-d-\alpha>1, \beta>d, m>d$.

Let $\left|S^{d-1}\right|$ denotes the $(d-1)$-dimentional Lebesgue measure of the unit sphere.

Theorem 6.1. Let $v, w_{1}$ satisfy the assumptions of Theorem 5.1, where $\alpha>0, \sigma-d-\alpha>1$. Suppose also that $v \in W^{m, 1}\left(\mathbb{R}^{d}\right)$, where $m>d$, and $w_{1}$ satisfies (94), where $\beta>d$. Let $v_{\text {appr }}$ be defined by (76)-(79), (96). Then:

$$
\begin{aligned}
& \left|v(x)-v_{\text {appr }}(x, E)\right| \leq A_{1} E^{-\gamma_{1}}+A_{2} E^{-\gamma_{2}}, x \in D, \\
& A_{1}=\frac{\left|S^{d-1}\right| C_{7}(m)}{(2 \tau)^{m-d}(m-d)}+(1+2 \tau)^{\beta}(2 \tau)^{d} c_{3} C_{3} \eta^{3} \mu\left(B_{1}\right), \\
& A_{2}=\frac{(1+2 \tau)^{\beta}(2 \tau)^{d}}{(2-2 \tau)^{\sigma-d-\alpha}} \mu\left(B_{1}\right) c_{3} C_{4} \eta^{2},
\end{aligned}
$$

where $\gamma_{1}, \gamma_{2}$ are given by (98), $C_{3}, C_{4}, C_{7}$ are given by (84), (85), (97), $\tau$ is the number of (96).

Theorem 6.2. Let $v, w_{1}$ satisfy the assumptions of Theorem 5.2, where $\alpha>0, \sigma-d-\alpha>1$. Suppose also that $v \in W^{m, 1}\left(\mathbb{R}^{d}\right)$, where $m>d$, and $w_{1}$ satisfies (94), where $\beta>d$. Let $v_{\text {appr }}$ be defined by (76)-(78), (80), (96). Then:

$$
\begin{aligned}
& \left|v(x)-v_{\text {appr }}(x, E)\right| \leq A_{3} E^{-\gamma_{1}}+A_{4} E^{-\gamma_{2}}, x \in D, \\
& A_{3}=\frac{\left|S^{d-1}\right| C_{7}(m)}{(2 \tau)^{m-d}(m-d)}+(1+2 \tau)^{\beta}(2 \tau)^{d} c_{3} C_{5} \eta^{3} \mu\left(B_{1}\right), \\
& A_{4}=\frac{(1+2 \tau)^{\beta}(2 \tau)^{d}}{(2-2 \tau)^{\sigma-d-\alpha}} \mu\left(B_{1}\right) c_{3} C_{6} \eta^{2},
\end{aligned}
$$

where $\gamma_{1}, \gamma_{2}$ are given by (98), $C_{5}, C_{6}, C_{7}$ are given by (87), (88), (97), $\tau$ is the number of (96).

The proofs of Theorems 6.1, 6.2 are given in Section 8. In these proofs we proceed from Theorems 5.1, 5.2, formula (96) and estimates (94), (97).

Remark 6.1. If the assumption that $v \in W^{m, 1}\left(\mathbb{R}^{d}\right), m>d$, is not fulfilled, then the considerations of the present section can be developed for apodized (smoothed) $v$ in a similar way with considerations of Section 6.1 of [15] and Theorem 3.2, Remark 3.3 of [16]. 


\section{Proofs of Theorems 5.1 and 5.2}

Recall that

$$
\mathcal{F}\left(\varphi_{1} \varphi_{2}\right)(p)=\left(\mathcal{F} \varphi_{1} * \mathcal{F} \varphi_{2}\right)(p)=\int_{\mathbb{R}^{d}} \mathcal{F} \varphi_{1}\left(p-p^{\prime}\right) \mathcal{F} \varphi_{2}\left(p^{\prime}\right) d p^{\prime}, p \in \mathbb{R}^{d}
$$

where $\varphi_{1}, \varphi_{2}$ are test functions.

\subsection{Proof of Theorem 5.1}

We consider

$$
\begin{aligned}
& \Delta h(p, E)=\left|\mathcal{F}\left(v+w_{1}\right)(p)\right|^{2}-h(p, E), p \in \mathbb{R}^{d}, \\
& \Delta u(x, E)=u(x)-u_{\text {appr }}(x, E), x \in \mathbb{R}^{d}, \\
& \Delta q(x, E)=q(x)-q_{a p p r}(x, E), x \in \mathbb{R}^{d}, \\
& \Delta \hat{v}(p, E)=\hat{v}(p)-\hat{v}_{\text {appr }}(p, E), p \in B_{(2-\delta) E},
\end{aligned}
$$

where $\hat{v}=\mathcal{F} v, q, u$ are the functions of $(32)-(34)$ with $\chi_{D-\Omega, \varepsilon}$ in place of $\chi_{D-\Omega}$, and $\hat{v}_{\text {appr }}$, $q_{a p p r}, u_{a p p r}, h$ are the functions of (76)-(79). We have that

$$
\begin{aligned}
& \Delta u(\cdot, E)=\mathcal{F}^{-1} \Delta h(\cdot, E), \Delta q(\cdot, E)=\chi_{D-\Omega, \varepsilon} \mathcal{F}^{-1} \Delta h(\cdot, E), \\
& \Delta \hat{v}(p, E)=\left(\overline{\mathcal{F} w_{1}}(p)\right)^{-1} \mathcal{F} \Delta q(p, E), \\
& \mathcal{F} \Delta q(p, E)=\int_{\mathbb{R}^{d}} \mathcal{F} \chi_{D-\Omega, \varepsilon}\left(p-p^{\prime}\right) \Delta h\left(p^{\prime}, E\right) d p^{\prime}, p \in B_{(2-\delta) \sqrt{E}},
\end{aligned}
$$

where in (112) we used (105) and (110).

First, we estimate $\Delta h$. Definition (106) can be rewritten as

$$
\begin{aligned}
& \Delta h(p, E)=\left|\mathcal{F}\left(v+w_{1}\right)(p)\right|^{2}-\left|f_{1}\left(k_{E}(p), l_{E}(p)\right)\right|^{2}, p \in B_{2 \sqrt{E}}, \\
& \Delta h(p, E)=\left|\mathcal{F}\left(v+w_{1}\right)(p)\right|^{2}-\left|\mathcal{F} w_{1}(p)\right|^{2}, p \in \mathbb{R}^{d} \backslash B_{2 \sqrt{E}} .
\end{aligned}
$$

Due to (18), (113), we have that

$$
|\Delta h(p, E)| \leq c(D \cup \Omega) \eta^{3} E^{-1 / 2}, p \in B_{2 \sqrt{E}}, \text { for } E^{1 / 2} \geq \rho(D \cup \Omega, \eta) .
$$

In addition, we have that

$$
|\Delta h(p, E)| \leq(2 \pi)^{-2 d} \eta^{2}\left(\mu(D)^{2}+2 \mu(D) \mu(\Omega)\right), p \in \mathbb{R}^{d} \backslash B_{2 \sqrt{E}} .
$$

Estimate (116) follows from the following estimates

$$
\begin{aligned}
& \left.|| \mathcal{F}\left(v+w_{1}\right)\right|^{2}-\left|\mathcal{F} w_{1}\right|^{2}|\leq|\left|\mathcal{F}\left(v+w_{1}\right)\right|-\left|\mathcal{F} w_{1}\right| \mid\left(\left|\mathcal{F}\left(v+w_{1}\right)\right|+\left|\mathcal{F} w_{1}\right|\right) \leq \\
& \leq|\mathcal{F} v|^{2}+2\left|\mathcal{F} v \| \mathcal{F} w_{1}\right| \\
& |\mathcal{F} v| \leq(2 \pi)^{-d} \eta \mu(D),\left|\mathcal{F} w_{1}\right| \leq(2 \pi)^{-d} \eta \mu(\Omega) .
\end{aligned}
$$

In (117) we used the inequalities

$$
-|a| \leq|a+b|-|b| \leq|a|, a=\mathcal{F} v, b=\mathcal{F} w_{1} .
$$

In view of $(111),(112)$, estimating $\Delta \hat{v}$ consists of the following. We have that

$$
\begin{aligned}
& |\mathcal{F} \Delta q(p, E)| \leq \int_{B_{2 \sqrt{E}}}\left|\mathcal{F} \chi_{D-\Omega, \varepsilon}\left(p-p^{\prime}\right)\right|\left|\Delta h\left(p^{\prime}, E\right)\right| d p^{\prime}+ \\
& +\int_{\mathbb{R}^{d} \backslash B_{2 \sqrt{E}}}\left|\mathcal{F} \chi_{D-\Omega, \varepsilon}\left(p-p^{\prime}\right)\right|\left|\Delta h\left(p^{\prime}, E\right)\right| d p^{\prime}=I_{1}(p, E)+I_{2}(p, E),
\end{aligned}
$$


where $p \in B_{(2-\delta) \sqrt{E}}$.

Using (81), (115) we estimate $I_{1}$ as follows:

$$
\begin{aligned}
& \left|I_{1}(p, E)\right| \leq c(D \cup \Omega) \eta^{3} E^{-1 / 2} \int_{B_{2 \sqrt{E}}}\left|\mathcal{F} \chi_{D-\Omega, \varepsilon}\left(p-p^{\prime}\right)\right| d p^{\prime}, \\
& \int_{B_{2 \sqrt{E}}}\left|\mathcal{F} \chi_{D-\Omega, \varepsilon}\left(p-p^{\prime}\right)\right| d p^{\prime} \leq \int_{\mathbb{R}^{d}}\left|\mathcal{F} \chi_{D-\Omega, \varepsilon}\left(p^{\prime}\right)\right| d p^{\prime} \leq \\
& \leq C_{1}\left(\sigma_{1}\right) \int_{\mathbb{R}^{d}} \frac{d p^{\prime}}{\left(1+\left|p^{\prime}\right|\right)^{\sigma_{1}}}=C_{1}\left(\sigma_{1}\right) C_{2}\left(\sigma_{1}\right), \sigma_{1}>d
\end{aligned}
$$

where $p \in B_{(2-\delta) \sqrt{E}}, C_{1}, C_{2}$ are the constants of (81), (82).

Using (81), (116) we estimate $I_{2}$ as follows:

$$
\begin{aligned}
& \left|I_{2}(p, E)\right| \leq(2 \pi)^{-2 d} \eta^{2}\left(\mu(D)^{2}+2 \mu(D) \mu(\Omega)\right) C_{1}(\sigma) \int_{\mathbb{R}^{d} \backslash B_{2 \sqrt{E}}} \frac{d p^{\prime}}{\left(1+\left|p-p^{\prime}\right|\right)^{\sigma}} \\
& \int_{\mathbb{R}^{d} \backslash B_{2 \sqrt{E}}} \frac{d p^{\prime}}{\left(1+\left|p-p^{\prime}\right|\right)^{\sigma}}=\int_{\mathbb{R}^{d} \backslash B_{2 \sqrt{E}}} \frac{d p^{\prime}}{\left(1+\left|p-p^{\prime}\right|\right)^{\sigma-d-\alpha}\left(1+\left|p-p^{\prime}\right|\right)^{d+\alpha}} \leq \\
& \leq \frac{1}{\left(1+\delta E^{1 / 2}\right)^{\sigma-d-\alpha}} \int_{\mathbb{R}^{d} \backslash B_{2 \sqrt{E}}} \frac{d p^{\prime}}{\left(1+\left|p-p^{\prime}\right|\right)^{d+\alpha}} \leq \\
& \leq \frac{1}{\left(1+\delta E^{1 / 2}\right)^{\sigma-d-\alpha}} \int_{\mathbb{R}^{d}} \frac{d p^{\prime}}{\left(1+\left|p-p^{\prime}\right|\right)^{d+\alpha}} \leq \frac{C_{2}(d+\alpha)}{\left(1+\delta E^{1 / 2}\right)^{\sigma-d-\alpha}},
\end{aligned}
$$

where $p \in B_{(2-\delta) \sqrt{E}}, \alpha>0, \sigma-d-\alpha>0, C_{1}, C_{2}$ are the constants of (81), (82).

Formulas (83)-(85) follow from (109), (111), (120)-(124).

Theorem 5.1 is proved.

\subsection{Proof of Theorem 5.2}

We consider

$$
\Delta h(p, E)=\left|\mathcal{F}\left(v+w_{1}\right)(p)\right|^{2}-|\mathcal{F} v(p)|^{2}-h(p, E), p \in \mathbb{R}^{d},
$$

where $h$ is defined in (80). We also consider $\Delta u, \Delta q, \Delta \hat{v}$ defined as in (107)-(109), where $u, q, \hat{v}=$ $\mathcal{F} v$ are defined by $(35)-(37), u_{\text {appr }}, q_{\text {appr }}, \hat{v}_{\text {appr }}$ are defined by (76)-(78), (80).

Note that formulas (110)-(112) remain valid with $\Delta h$ given by (125).

First, we estimate $\Delta h$. Definition (125) can be rewritten as

$$
\begin{aligned}
& \Delta h(p, E)=\left(\left|\mathcal{F}\left(v+w_{1}\right)(p)\right|^{2}-\left|f_{1}\left(k_{E}(p), l_{E}(p)\right)\right|^{2}\right)-\left(|\mathcal{F} v(p)|^{2}-\left|f\left(k_{E}(p), l_{E}(p)\right)\right|^{2}\right), \\
& p \in B_{2 \sqrt{E}}, \\
& \Delta h(p, E)=\left|\mathcal{F}\left(v+w_{1}\right)(p)\right|^{2}-|\mathcal{F} v(p)|^{2}-\left|\mathcal{F} w_{1}(p)\right|^{2}, p \in \mathbb{R}^{d} \backslash B_{2 \sqrt{E}} .
\end{aligned}
$$

Due to (18), (126), we have that

$$
\begin{aligned}
& |\Delta h(p, E)| \leq(c(D \cup \Omega)+c(D)) \eta^{3} E^{-1 / 2}, p \in B_{2 \sqrt{E}}, \\
& \text { for } E^{1 / 2} \geq \rho(D \cup \Omega, \eta) .
\end{aligned}
$$

In addition, we have that

$$
|\Delta h(p, E)| \leq 2(2 \pi)^{-2 d} \eta^{2} \mu(D) \mu(\Omega), p \in \mathbb{R}^{d} \backslash B_{2 \sqrt{E}} .
$$

Estimate (129) follows from (118) and the following estimate

$$
\left.|| \mathcal{F}\left(v+w_{1}\right)\right|^{2}-|\mathcal{F} v|^{2}-\left|\mathcal{F} w_{1}\right|^{2}|\leq 2| \mathcal{F} v|| \mathcal{F} w_{1} \mid
$$


Estimate (120) for $\mathcal{F} \Delta q$ remains valid with $\Delta h$ given by (125).

In addition: using (128) we have that

$$
\left|I_{1}(p, E)\right| \leq(c(D)+c(D \cup \Omega)) \eta^{3} E^{-1 / 2} \int_{B_{2 \sqrt{E}}}\left|\mathcal{F} \chi_{D-\Omega, \varepsilon}\left(p-p^{\prime}\right)\right| d p^{\prime}, p \in B_{(2-\delta) \sqrt{E}}
$$

using (129) we have that

$$
\left|I_{2}(p, E)\right| \leq 2(2 \pi)^{-2 d} \eta^{2} \mu(D) \mu(\Omega) C_{1}(\sigma) \int_{\mathbb{R}^{d} \backslash B_{2 \sqrt{E}}} \frac{d p^{\prime}}{\left(1+\left|p-p^{\prime}\right|\right)^{\sigma}}, p \in B_{(2-\delta) \sqrt{E}}, \sigma>d .
$$

Formulas (86)-(88) follow from (109), (111), (120), (122), (124), (131), (132).

Theorem 5.2 is proved.

\section{Proof of Theorems 6.1 and 6.2}

The following formulas hold:

$$
\begin{aligned}
& v(x)=\int_{\mathbb{R}^{d}} e^{-i x p} \hat{v}(p) d p, \quad v_{a p p r}(x, E)=\int_{B_{(2-\delta) \sqrt{E}}} e^{-i x p} \widehat{v}_{a p p r}(p, E) d p, x \in D, \\
& \left|v(x)-v_{a p p r}(x, E)\right| \leq \int_{B_{(2-\delta) \sqrt{E}}}\left|\hat{v}(p)-\hat{v}_{a p p r}(p, E)\right| d p+\int_{\mathbb{R}^{d} \backslash B_{(2-\delta) \sqrt{E}}}|\hat{v}(p)| d p,
\end{aligned}
$$

where in (133) we used the inversion formula for the Fourier transform and the definition of $v_{\text {appr }}$.

Recall that according to (96) we have that

$$
(2-\delta(E)) \sqrt{E}=2 \tau E^{\gamma}, \gamma=\frac{1}{2} \frac{1}{m+\beta} .
$$

Using (97), (135) we have that

$$
\begin{aligned}
& I_{0}:=\int_{\mathbb{R}^{d} \backslash B_{(2-\delta) \sqrt{E}}}|\hat{v}(p)| d p \leq C_{7}(m)\left|S^{d-1}\right| \int_{(2-\delta) \sqrt{E}}^{\infty} \frac{d r}{r^{m-d+1}}= \\
& =\frac{C_{7}(m)\left|S^{d-1}\right|}{(m-d)((2-\delta) \sqrt{E})^{m-d}}=\frac{C_{7}(m)\left|S^{d-1}\right|}{(m-d)(2 \tau)^{m-d}} E^{-\gamma(m-d)} .
\end{aligned}
$$

From Theorem 5.1 we have that

$$
\left|\hat{v}(p)-\hat{v}_{a p p r}(p, E)\right| \leq\left|\hat{w}_{1}(p)\right|^{-1}\left(C_{3} E^{-1 / 2} \eta^{3}+\frac{C_{4} \eta^{2}}{\left(1+\delta E^{1 / 2}\right)^{\sigma-d-\alpha}}\right), p \in B_{(2-\delta) \sqrt{E}} .
$$

From (94), (135) and (137) we obtain

$$
\begin{aligned}
& \int_{B_{(2-\delta) \sqrt{E}}}\left|\hat{v}(p)-\hat{v}_{a p p r}(p, E)\right| d y \leq c_{3} \int_{B_{(2-\delta) \sqrt{E}}}(1+|p|)^{\beta}\left(C_{3} \eta^{3} E^{-1 / 2}+\frac{C_{4} \eta^{2}}{\left(1+\delta E^{1 / 2}\right)^{\sigma-d-\alpha}}\right) d p= \\
& =c_{3}\left(C_{3} \eta^{3} I_{1}+C_{4} \eta^{2} I_{2}\right), \\
& I_{1}:=E^{-1 / 2} \int_{B_{(2-\delta) \sqrt{E}}}(1+|p|)^{\beta} d p \leq E^{-1 / 2}(1+(2-\delta) \sqrt{E})^{\beta} \mu\left(B_{1}\right)((2-\delta) \sqrt{E})^{d} \leq \\
& \leq E^{-1 / 2+d / 2}(2-\delta)^{d}(1+(2-\delta) \sqrt{E})^{\beta} \mu\left(B_{1}\right)=E^{-1 / 2}(2 \tau)^{d} E^{\gamma d}\left(1+2 \tau E^{\gamma}\right)^{\beta} \mu\left(B_{1}\right) \\
& I_{2}:=\frac{\int_{B_{(2-\delta) \sqrt{E}}}(1+|p|)^{\beta} d p}{\left(1+\delta E^{1 / 2}\right)^{\sigma-d-\alpha}} \leq \frac{(1+(2-\delta) \sqrt{E})^{\beta}(2-\delta)^{d} E^{d / 2} \mu\left(B_{1}\right)}{\left(1+\delta E^{1 / 2}\right)^{\sigma-d-\alpha}}= \\
& =\frac{\left(1+2 \tau E^{\gamma}\right)^{\beta}(2 \tau)^{d} E^{\gamma d} \mu\left(B_{1}\right)}{\left(1+\delta E^{1 / 2}\right)^{\sigma-d-\alpha}} .
\end{aligned}
$$


Using (135), (139), (140), for $E \geq 1$ as in (96), we have that

$$
\begin{aligned}
& I_{1} \leq(1+2 \tau)^{\beta} \mu\left(B_{1}\right)(2 \tau)^{d} E^{-1 / 2+\gamma d+\gamma \beta} \\
& I_{2} \leq \frac{(1+2 \tau)^{\beta}(2 \tau)^{\beta} \mu\left(B_{1}\right) E^{\gamma \beta+\gamma d}}{\left(\delta E^{1 / 2}\right)^{\sigma-d-\alpha}} \leq \frac{(1+2 \tau)^{\beta}(2 \tau)^{\beta} \mu\left(B_{1}\right) E^{\gamma \beta+\gamma d}}{\left(2 \sqrt{E}\left(1-\tau E^{\gamma-1 / 2}\right)\right)^{\sigma-d-\alpha}} \leq \\
& \leq \frac{(1+2 \tau)^{\beta}(2 \tau)^{\beta} \mu\left(B_{1}\right) E^{\gamma \beta+\gamma d}}{2^{\sigma-d-\alpha} E^{(\sigma-d-\alpha) / 2}\left(1-\tau E^{\gamma-1 / 2}\right)^{\sigma-d-\alpha}} \leq \frac{(1+2 \tau)^{\beta}(2 \tau)^{\beta} \mu\left(B_{1}\right) E^{\gamma \beta+\gamma d}}{2^{\sigma-d-\alpha} E^{(\sigma-d-\alpha) / 2}(1-\tau)^{\sigma-d-\alpha}}
\end{aligned}
$$

where in the last inequality we used that $\gamma<1 / 2$.

In addition, taking into account the value of $\gamma$ we have that, for $E \rightarrow+\infty$ :

$$
\begin{aligned}
& I_{0}=\mathcal{O}\left(E^{-\frac{1}{2} \frac{m-d}{m+\beta}}\right)=\mathcal{O}\left(E^{-\gamma_{1}}\right), \\
& I_{1}=\mathcal{O}\left(E^{-\frac{1}{2}+\frac{1}{2} \frac{d+\beta}{m+\beta}}\right)=\mathcal{O}\left(E^{-\frac{1}{2} \frac{m-d}{m+\beta}}\right)=\mathcal{O}\left(E^{-\gamma_{1}}\right), \\
& I_{2}=\mathcal{O}\left(E^{\frac{1}{2} \frac{\beta+d}{\beta+m}-\frac{\sigma-d-\alpha}{2}}\right)=\mathcal{O}\left(E^{-\frac{\sigma-d-\alpha-1}{2}}\right)=\mathcal{O}\left(E^{-\gamma_{2}}\right) .
\end{aligned}
$$

Estimate (99) follows from formulas (134), (136), (138), (141)-(145).

Theorem 6.1 is proved.

The proof of Theorem 6.2, proceeding from formula (96) and Theorem 5.2, is similar to the proof of Theorem 6.1, proceeding from formula (96) and Theorem 5.1.

\section{Acknowledgement}

V.N. Sivkin was partially supported by Russian Science Foundation, RSF, grant No 20-11-20261.

\section{References}

1. T. Aktosun, P. E. Sacks, Inverse problem on the line without phase information, Inverse Problems 14, 211-224 (1998)

2. A.D. Agaltsov, T. Hohage, R.G. Novikov, An iterative approach to monochromatic phaseless inverse scattering, Inverse Problems 35, 24001 (24 pp.) (2019)

3. A. D. Agaltsov, R.G. Novikov, Error estimates for phaseless inverse scattering in the Born approximation at high energies, J. Geom. Anal. (2017), https://doi.org/10.1007/s12220-017-9872-6, e-print: https://hal.archives-ouvertes.fr/hal-01303885v2

4. A.H. Barnett, Ch.L. Epstein, L.F. Greengard, J.F. Magland, Geometry of the phase retrieval problem, Inverse Problems 36, 094003 (2020)

5. F.A. Berezin, M.A. Shubin, The Schrödinger Equation, Mathematics and Its Applications, Vol. 66, Kluwer Academic, Dordrecht, 1991

6. M. Born, Quantenmechanik der Stossvorgange, Zeitschrift fur Physik 38 (11-12), 803-827 (1926)

7. K. Chadan, P.C. Sabatier, Inverse Problems in Quantum Scattering Theory, 2nd edn. Springer, Berlin, 1989

8. K. Engel, B. Laasch, The modulus of the Fourier transform on a sphere determines 3-dimensional convex polytopes, J. Inverse Ill-Posed Probl., https://doi.org/10.1515/jiip-2020-0103

9. G. Eskin, Lectures on Linear Partial Differential Equations, Graduate Studies in Mathematics, Vol. 123, American Mathematical Society, 2011

10. L.D. Faddeev, Uniqueness of the solution of the inverse scattering problem, Vestn. Leningrad Univ. 7, 126-130 (1956) (in Russian)

11. L.D. Faddeev, S.P. Merkuriev, Quantum Scattering Theory for Multi-particle Systems, Mathematical Physics and Applied Mathematics, 11. Kluwer Academic Publishers Group, Dordrecht, 1993 
12. A.A. Govyadinov, G.Y. Panasyuk, J.C. Schotland, Phaseless three-dimensional optical nanoimaging, Phys. Rev. Lett. 103, 213901 (2009)

13. T. Hohage, R.G. Novikov, Inverse wave propagation problems without phase information, Inverse Problems 35, 070301 (4 pp.)(2019)

14. O. Ivanyshyn, R. Kress, Inverse scattering for surface impedance from phase-less far field data, J. Comput. Phys. 230(9), 3443-3452 (2011)

15. M. Isaev, R.G. Novikov, Hölder-logarithmic stability in Fourier synthesis, Inverse Problems 36, 125003 (2020)

16. M. Isaev, R.G. Novikov, Stability estimates for reconstruction from the Fourier transform on the ball, J. Inverse Ill-Posed Probl., https://doi.org/10.1515/jiip-2020-0106

17. M.V. Klibanov, Phaseless inverse scattering problems in three dimensions, SIAM J.Appl. Math. 74(2), 392-410 (2014)

18. M.V. Klibanov, N.A. Koshev, D.-L. Nguyen, L.H. Nguyen, A. Brettin, V.N. Astratov, A numerical method to solve a phaseless coefficient inverse problem from a single measurement of experimental data, SIAM J. Imaging Sci. 11(4), 2339-2367 (2018)

19. M.V. Klibanov, V.G. Romanov, Reconstruction procedures for two inverse scattering problems without the phase information, SIAM J. Appl. Math. 76(1), 178-196 (2016)

20. M.V. Klibanov, P.E. Sacks, A.V. Tikhonravov, The phase retrieval problem, Inverse Problems 11, 1-28 (1995)

21. B. Leshem et al., Direct single-shot phase retrieval from the diffraction pattern of separated objects, Nature Communications 7(1), 1-6 (2016)

22. R. G. Novikov, An iterative approach to non-overdetermined inverse scattering at fixed energy, Sbornik: Mathematics 206(1), 120-134 (2015)

23. R. G. Novikov, Inverse scattering without phase information, Seminaire Laurent Schwartz - EDP et applications (2014-2015), Exp. No16, 13p

24. R. G. Novikov, Explicit formulas and global uniqueness for phaseless inverse scattering in multidimensions, J. Geom. Anal. 26(1), 346-359 (2016), e-print: https://hal.archives-ouvertes.fr/hal-01095750v1

25. R. G. Novikov, Multipoint formulas for phase recovering from phaseless scattering data, J. Geom. Anal., https://doi.org/10.1007/s12220-019-00329-6

26. R.G. Novikov, Multidimensional inverse scattering for the Schrödinger equation, Book series: Springer Proceedings in Mathematics and Statistics. Title of volume: Mathematical Analysis, its Applications and Computation - ISAAC 2019, Aveiro, Portugal, July 29-August 2; Editors: P. Cerejeiras, M. Reissig (to appear), e-preprint: https://hal.archives-ouvertes.fr/hal-02465839v1

27. R. G. Novikov, V. N. Sivkin, Error estimates for phase recovering from phaseless scattering data, Eurasian Journal of Mathematical and Computer Applications, vol. 8(1), 44-61, (2020)

28. S.G. Podorov, K.M. Pavlov, D.M. Paganin, A non-iterative reconstruction method for direct and unambiguous coherent diffractive imaging, Optics Express, 15(16), 9954-9962 (2007)

29. V. G. Romanov, Inverse problems without phase information that use wave interference, Sib. Math. J. 59(3), 494-504 (2018)

30. V.G. Romanov, A phaseless inverse problem for electrodynamic equations in the dispersible medium, Applicable Analysis, https://doi.org/10.1080/00036811.2020.1846721

31. X. Xu, B. Zhang, H. Zhang, Uniqueness in inverse electromagnetic scattering problem with phaseless far-field data at a fixed frequency, IMA Journal of Applied Mathematics 85(6), 823-839 (2020) 